\title{
Consecuencias ambientales y socioeconómicas del comercio de los peces ornamentales en Colombia
}

Environmental and socioeconomic consequences of ornamental fish trade in Colombia

Citar como: Moncaleano Gómez, E. M. y Franco-Ortega, J. A. (2020). Consecuencias ambientales y socioeconómicas del comercio de los peces ornamentales en Colombia. CITAS, 6(1). https://doi.org/10.15332/24224529.6367

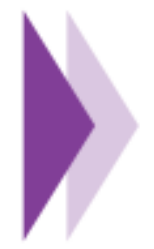

Erika Marcela Moncaleano Gómez ${ }^{1}$; Julio Alejandro Franco-Ortega ${ }^{2}$

1 Universidad Santo Tomás

2 Universidad Agraria de Colombia

Correo electrónico: erikamoncaleano@ustadistancia.edu.co alfranco12@gmail.com 


\section{Resumen}

El presente trabajo aborda una revisión de la literatura científica sobre las principales consecuencias ambientales y socioeconómicas del mercado de los peces ornamentales (PO) en Colombia. Como principales resultados se encontró que este comercio continúa siendo una actividad de intercambio mundial de especies exóticas que genera grandes ganancias. La extracción de estas especies causa desbalance en las poblaciones naturales que habitan los cuerpos de agua. Esto genera preocupaciones como el agotamiento del recurso asociado con la sobrepesca y prácticas de extracción, la introducción de especies exóticas a los distintos lugares de destino, los factores de estrés propios de los animales que han provocado riesgos emergentes al ser portadores de patógenos como virus y bacterias. Adicionalmente, el uso indiscriminado de compuestos químicos como sales, antibióticos, fármacos, entre otros, en la recepción de los peces y en los cultivos genera cargas químicas contaminantes del recurso hidrico. Sumado a lo anterior, las malas prácticas de alimentación conducen al deterioro de la calidad del agua, lo que causa impactos adicionales que deben ser considerados. Esta actividad ha contribuido al desarrollo socioeconómico y bienestar en las comunidades pesqueras que dependen de manera directa e indirecta de este recurso. Se identificaron problemáticas relacionadas con la pobreza en las comunidades, el bajo nivel educativo, la falta de oportunidades, el abandono del Estado y el poco incentivo al cultivo que impiden el desarrollo de esta actividad, más allá de la recolección.

La actividad comercial del acuarismo se practica globalmente, esta distribuye gran cantidad de organismos como peces, invertebrados, plantas marinas y de agua dulce a los principales importadores del comercio de peces ornamentales como Estados Unidos, la Unión Europea y Japón (Stevens et ál. 2017). También es considerada como la actividad de mayor intercambio de animales a nivel mundial (Ploeg, 2007). Se estima que involucra más de dos millones de personas, tanto directa como indirectamente (incluidos los aficionados) (Domínguez y Bottella, 2014), y es considerada de alto valor (Organización de las Naciones Unidas para la Alimentación y la Agricultura [FAO], 2016).

En Colombia se comercializan cerca de 25 millones de peces ornamentales (PO), donde el $88 \%$ de los peces comercializados provienen de la cuenca de la Orinoquía; el $10 \%$, de la cuenca Amazónica, el $1.7 \%$, de la cuenca del Pacífico, y el $0.3 \%$, de la cuenca del Magdalena (Prada-Pedreros et ál, 2009; Zúniga, 2010). Estos, en su mayoría, son extraídos del medio natural y comercializados con fines de exportación. Sin embargo, es una actividad que divide las opiniones (Raghavan et ál., 2013; King, 2019), especialmente en términos de los efectos ambientales y sostenibilidad a largo plazo. Más allá de la extracción, es un importante contribuyente para las economías locales que dependen del recurso para su subsistencia.

\section{Materiales o recursos}

Para identificar las consecuencias ambientales y socioeconómicas del comercio de los peces ornamentales en Colombia, se planteó un método de consulta de revisión de literatura rápida, con el fin de proporcionar una evaluación de los principales impactos, mediante el uso de métodos de revisión sistemática para buscar y evaluar críticamente la investigación existente (Grant y Booth, 2009). A partir de la revisión rápida se realizó un análisis de
Palabras clave: peces ornamentales, impactos, ambientales, socioeconómico, Colombia.

Abstract

This paper makes a review of the scientific literature on the main environmental and socioeconomic consequences of the ornamental fish market (OF) in Colombia. As main results, it was found that this trade continues to be an activity of global exchange of exotic species that generates large profits. The extraction of these species causes imbalance in the natural populations that inhabit water bodies. This raises concerns such as resource depletion associated with overfishing and extraction practices, the introduction of exotic species to the different destinations and stress factors to the animals that have caused emerging risks as carriers of pathogens such as viruses and bacteria. Additionally, the indiscriminate use of chemical compounds such as salts, antibiotics, medicines, among others, in the reception of fish and crops generates chemical loads that contaminate water resources. Furthermore, poor feeding practices lead to the deterioration of water quality, which causes additional impacts that must be considered. This activity has contributed to the socioeconomic development and well-being of the fishing communities that depend directly and indirectly on this resource. Problems related to poverty in the communities, low education level, lack of opportunities, neglect of the State and little incentives for farming were identified, which prevent the development of this activity, beyond harvesting.

Keywords: ornamental fishes, impacts, environmental, socioeconomic, Colombia.

reflexión con la finalidad de dar a conocer los impactos ambientales y socioeconómicos de los peces ornamentales.

Se realizó la búsqueda de literatura disponible en buscadores como Google Scholar y Redalyc, además de bases de datos como Web of Sciencie, Scopus, ScienceDirect y Scielo. Se utilizaron las siguientes palabras claves: 'ornamental fish', 'peces Colombia', 'fish wild', 'impactos ambientales acuicultura', 'freshwater aquarium', 'pesca artesanal', 'comercio peces ornamentales', 'comunidades pesqueras'. La búsqueda se realizó en el idioma español e inglés utilizando las posibles combinaciones dadas por las palabras clave. La revisión tuvo en cuenta artículos científicos, informes o reportes, y libros o cartillas institucionales.

\section{Resultados y discusión}

\section{Consecuencias ambientales}

El comercio de PO genera un desbalance en las poblaciones naturales que habitan los ríos, quebradas, mares y demás cuerpos de agua donde son extraídos, para posteriormente ser comercializados. En general los pescadores o colectores de corales y de peces realizan sus actividades como depredadores, que apuntan tanto a especies abundantes como raras. Estas últimas no serían viables para el comercio porque son de difícil acceso y, por lo tanto, no pueden satisfacer una constante demanda del mercado; además, son muy sensibles a su manipulación y transporte, por lo que quedan en riesgo de amenaza o en peligro ecológico y son particularmente vulnerables (Rhyne et ál., 2009; Rhyne et ál., 2012 y Raghavan et ál., 2013).

Actualmente los ecosistemas acuáticos donde habitan las especies de uso ornamental sufren un deterioro ocasionado por la contaminación debido al aporte de aguas servidas de los centros urbanos y a actividades como la construcción, la minería legal 
e ilegal, sumado a la desecación de los cauces por la extracción excesiva de agua, principalmente en los distritos de riego para la agricultura, cultivos de palma africana, aprovechamiento forestal, ampliación de la frontera agrícola, entre otros (Barato, 2012; Ortega-Lara, 2016).

Es así como el comercio de PO introduce miles de especies exóticas anualmente a diferentes partes del mundo, y un número importante ha ocupado ambientes de agua dulce y marinos para establecerse, reproducirse rápidamente y constituirse, en algunos casos, como invasores (Salazar et ál., 2008, Stern et ál., 2018). Estas introducciones de especies alóctonas a los ecosistemas por prácticas accidentales, intencionales o por escapes en su cultivo, trae consigo mayor competencia y amenaza, lo cual se traduce en alteraciones para las cadenas tróficas (Borja, 2002), trasmisión de patógenos o enfermedades como el virus del herpes Koi (Ladisa et ál., 2017), el virus del lago tilapia (TiLV) (Jaemwimol et ál., 2018, Phitchaya et ál., 2018), entre otros (Ito et ál., 2017); o ser vectores o trasmisores de parásitos, como el caso del Gyrodactylus cichlidarum en los poecílidos (Cyprinodontiformes) (GarcíaVásquez, Razo-Mendivil y Rubio-Godoy, 2017), los reportados por Dewi y colaboradores (2018) y bacterias (Passantino et ál., 2008, Hoshino et ál., 2018).

Esta problemática de introducción de especies afecta especialmente a países de gran diversidad íctica como Colombia (Ramírez et ál., 2001), Brasil (Monteiro-Neto et ál., 2003; Gasparini et ál., 2004), Perú (Sanna-Kaisa y Jukka, 2004) y Venezuela (FAO, 2005; Salazar et ál., 2008; Cabrera, 2005), donde sus prácticas de extracción son diversas: uso de dinamita, cianuro de sodio o quinaldina, para aturdir y capturar PO (Domínguez y Botella, 2014; Vaz et ál., 2017). Estas malas prácticas afectan las especies de interés y el hábitat circundante. El uso del cianuro y la quinaldina es ilegal en la mayoría de los países latinoamericanos; sin embargo, en algunos países de la región de Asia, como en Filipinas e Indonesia, se utiliza (Wood, 2001). El efecto de estos productos químicos nocivos en el hábitat sigue siendo desconocido, adicionalmente el uso de explosivos como la dinamita deben ser evaluados (Domínguez y Botella, 2014).

Otros impactos relacionados con la producción y mantenimiento en bodegas para la comercialización o exportación son los compuestos químicos formulados con sales cuaternarias de amonio (QAC), fármacos (antiparasitarios y antibióticos) y los compuestos químicos inorgánicos (azul de metileno, verde malaquita, permanganato de sodio), entre otros. Los QAC actúan como detergentes catiónicos, con funciones antimicrobianas; en la acuicultura estas cumplen la función de bactericidas y fungicidas (Gräslund y Bengtsson, 2001) y varían su actividad dependiendo de la longitud de la cadena de carbonos que componen su estructura química. Las QAC son tóxicas a concentraciones de $\mu \mathrm{g} / \mathrm{mL}$ (PPM) o menos para organismos acuáticos como algas, peces, moluscos, percebes, rotíferos, estrellas de mar y camarones.

Los antibióticos son usados regularmente como tratamiento preventivo (Buschmann y Fortt, 2005; Cabello, 2006), tanto en grandes bodegas comercializadoras como por los cultivadores. Estos compuestos, como las tetraciclinas, modifican la flora bacteriana del ambiente y su uso indiscriminado genera resistencias en las bacterias, lo que hace más difíciles y costosos los tratamientos en caso de enfermedad, además de causar efectos tóxicos en los organismos salvajes, alteraciones en la biodiversidad, incidencia en las cadenas tróficas y desarrollo de defensas antibacterianas en patógenos de los peces (Borja, 2002). En este sentido, la Autoridad Nacional de Pesca y
Acuicultura (Aunap), en el Plan Nacional para el Desarrollo de la Acuicultura Sostenible en Colombia 2014, evidencia debilidades y preocupaciones en relación con el uso indiscriminado de antibióticos, especialmente en la producción de PO.

El verde malaquita y el azul de metileno se utilizan constantemente para combatir hongos, parásitos y algunos microorganismos patógenos, debido a que no todos los productores o acuaristas conocen los tratamientos alternativos con sustancias menos contaminantes que reemplacen las acción de estos compuestos inorgánicos (Buschmann y Fortt, 2005). Sin embargo, su uso está restringido en Colombia por el daño ecológico que produce y sus efectos acumulativos y cancerígenos (Arboleda, 2006; Xu et ál., 2018).

Aunado a esto, en Colombia existe un desconocimiento relacionado con la biología básica, la nutrición y la alimentación, así como las exigencias nutricionales de las especies. En la práctica, esto conlleva a que alimentos formulados para peces de consumo como la tilapia (Oreochromis spp) y la trucha (Oncorhynchus mykiss) sean usados en la alimentación de los PO, lo que causa una extrapolación de requerimientos nutricionales entre especies (Velazco y Gutiérrez, 2019). Al desconocer los requerimientos, los PO deben ser adaptados al consumo de alimento artificial, lo cual es identificado como impacto ambiental negativo (Vergara et ál., 2005). Adicionalmente los alimentos comerciales para PO nacionales son genéricos, situación que ocasiona que los productores compren alimento importado específico para los hábitos alimenticios y requerimientos de algunas especies, lo cual aumenta los costos de producción por su alto valor.

De igual manera, en los centros de cultivo especializados suministran a los PO tanto alimento comercial para tilapia y trucha, como proporciones de proteína cruda, que está entre el $30 \%$ y el $47 \%$; consumido este alimento, se estima que el $75 \%$ de nitrógeno y fósforo se pierden, ya sea a través del alimento que no se consume, o por heces fecales y branquias (Folke y Kautsky, 1989; Buschmann et ál., 1996). Lo anterior, además de inducir el incremento de materia orgánica y la eutrofización de un ecosistema, genera que se incorporen ingredientes externos como el cobre, usado para combatir los organismos no deseados como las algas. Estos cambios afectan la diversidad y causan desequilibrios en las cadenas tróficas (Buschmann y Fortt, 2005).

Tales impactos se pueden detectar en un radio de un kilómetro alrededor del cultivo, los cuales están acumulados generalmente en el fondo acuático, donde se incrementa la demanda de oxígeno, la producción de sedimentos anóxicos y de gases tóxicos. Todo esto genera cambios en las comunidades bióticas y afecta la diversidad de los organismos bentónicos (Borja, 2002) que habitan el fondo acuático y constituyen uno de los eslabones más importantes en el flujo de materia y energía de los ecosistemas (Moreno et ál., 2017; Morales y García 2016).

Otras afectaciones ambientales se deben en menor escala al número de recambios periódicos de agua, desinfección y lavado de acuarios, tanques, estanques, cosechas, abono de estanques, mortalidad e interacciones entre organismos (Espinosa y Bermúdez, 2012). La falta de desarrollo tecnológico \#como en la transición al cultivo y mantenimiento en los sistemas de recirculación acuícola (RAS, por sus siglas en inglés)\# debe ser considerada en términos de aprovechamiento y uso de agua, pues se estima que permite reciclar hasta más del $90 \%$ del agua usada (Badiola, 2012) (figura 1). 
Figura 1. Impactos ambientales del comercio de PO

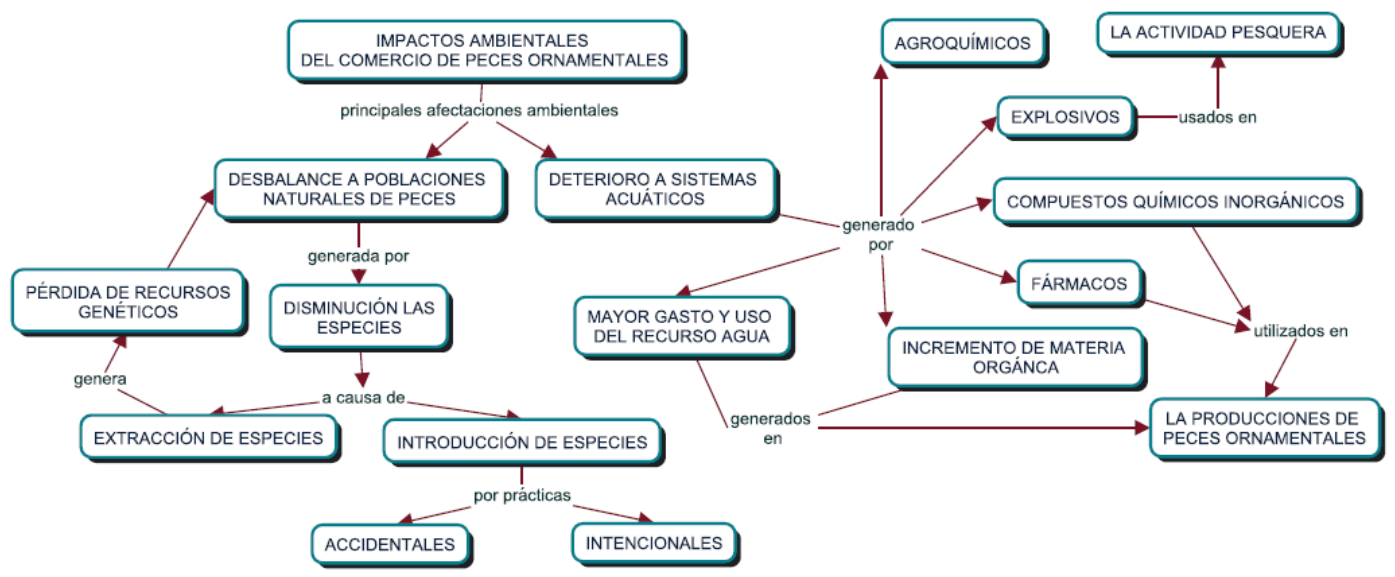

Fuente: elaboración propia.

\section{Consecuencias socioeconómicas}

La pesca artesanal ornamental apunta a la generación de empleos y beneficios económicos en las comunidades; dentro de esta actividad participan hombres, mujeres y niños de tiempo completo y parcial, en tareas directas e indirectas (Germain et ál., 2015), lo que contribuye al bienestar y al desarrollo social en las comunidades costeras (Denommee-Patriganni, 2011) y rivereñas (Chao y Prang, 1997). Estos colectivos de pescadores dependen, para su sustento, de los recursos acuáticos, especialmente en los países en vías de desarrollo como Colombia (FAO, 2005; 2012).

Los pescadores suelen trabajar solos o con miembros de una misma familia o comunidad. Si bien reconocen esta actividad como principal, se han visto afectados en su economía, en primer lugar, por la escasez del recurso; por lo tanto, deben gastar más tiempo y dinero en el desplazamiento para ubicar nuevos sitios para la pesca. Esto implica el uso de trasporte en moto en la cuenca de la Orinoquía y el uso de lanchas con motor en la cuenca amazónica. En segundo lugar, se ven afectados por la disminución de las ventas a causa del tráfico y la importación de PO. Es así como se ha incrementado el retiro de personas de este negocio, con la subsecuente generación de conflictos sobre la distribución de ingresos y oportunidades de empleo, asimismo afecta la realidad socioeconómica de la comunidad (FAO, 2005; Ajiaco-Martínez et ál., 2012; Guzmán-Maldonado y Lasso, 2014) que la obliga a dejar su medio de vida y buscar otras alternativas fuera de su entorno.

En Puerto Inírida, región de la Amazonia colombiana, se identificó que las comunidades de pescadores que están involucradas en la explotación de los recursos naturales tienen un nivel educativo medio ( $5 .^{\circ}$ de primaria) y dependen totalmente de los ingresos obtenidos de estas actividades de explotación para su subsistencia (Guerrero et ál., 2017). En consecuencia, este grado de escolaridad tan bajo y la dependencia del recurso imposibilitan en gran medida la diversificación de alternativas a la extracción y la probabilidad de prácticas responsables de cultivo que podrían promover el crecimiento económico local.

Las problemáticas asociadas con la degradación de la naturaleza, la falta de oportunidades y el abandono del Estado hacen que las comunidades caigan en la llamada trampa de la pobreza y medioambiente (figura 2). De igual manera limita el acceso a otros ingresos, y por estas razones son propensos a un círculo vicioso de la sobreexplotación y una mayor degradación del ambiente (Sobhee 2004; Barbier, 2010). Adicional a esto, la competencia por los recursos degrada aún más estas sociedades. 
Figura 2. Círculo vicioso de la sobreexplotación, degradación

del ambiente y pobreza relacionada con el comercio de PO

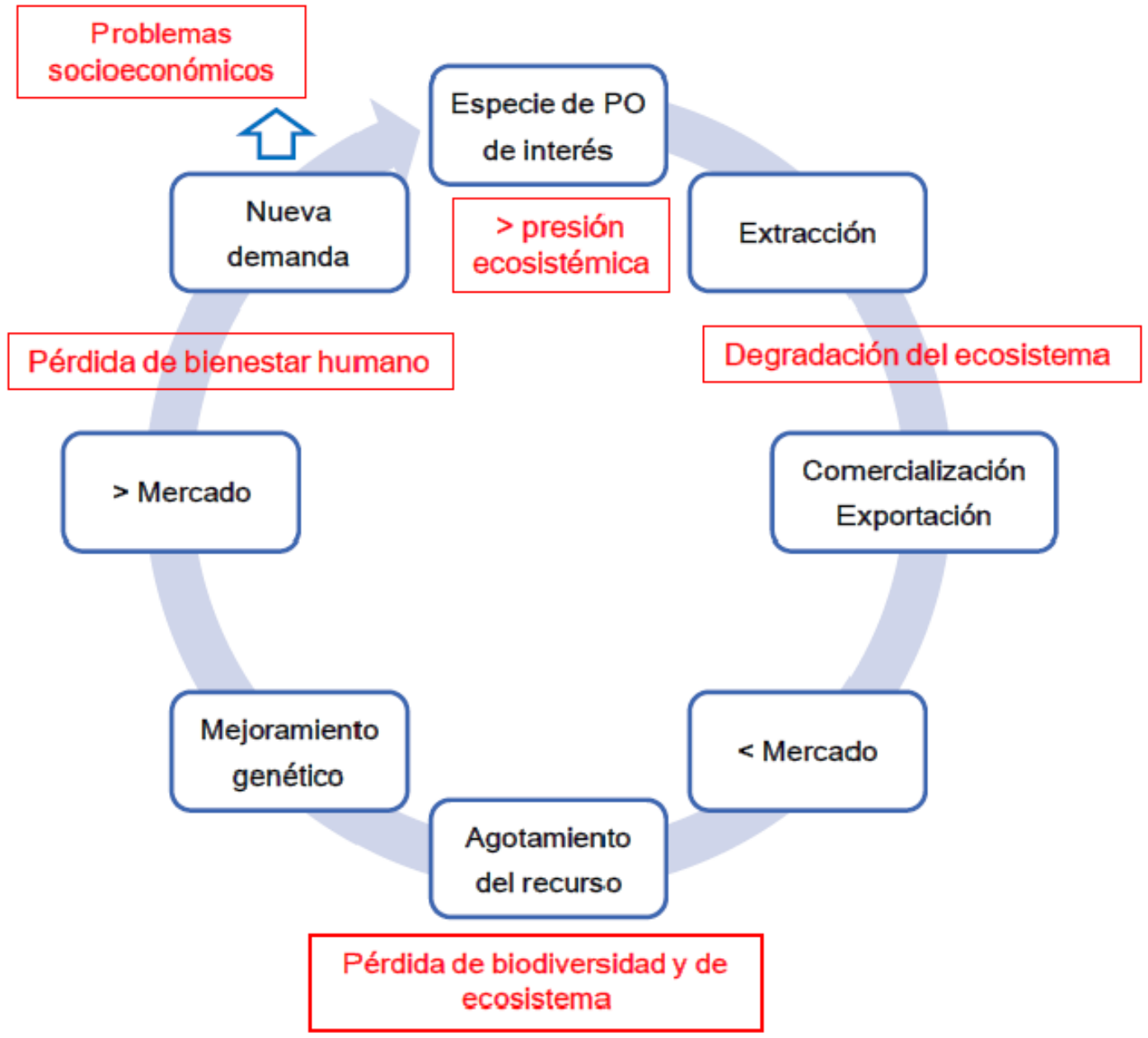

Fuente: elaboración propia.

También existen obstáculos, particularmente para los productores y sus iniciativas de fomentar el cultivo en cautiverio, pues su comercio es el más castigado y menos lucrativo; los mayores ingresos de esta actividad están concentrados en los comercializadores y finalmente en los exportadores (Mancera y Álvarez 2008; Rossoni et ál., 2014). De otra parte, hay países que reproducen especies nacionales en cautiverio y a nivel industrial (Chequia, Taiwán y Singapur), lo que para Colombia influye negativamente en las exportaciones y en la conservación de los recursos genéticos. Esto estimula la búsqueda de alternativas, como sustituir especies tradicionales por especies nuevas en cantidades reducidas con altos precios (Ortega-Lara, 2016), que son obtenidas de la pesca y no de la producción. Esto ocasiona de nuevo que la genética de las especies sea tratada y mejorada en otros países, lo cual fomenta un proceso repetitivo de pérdida del material genético de la biodiversidad del país (figura 3). 
Figura 3. Impactos socioeconómicos del comercio de PO

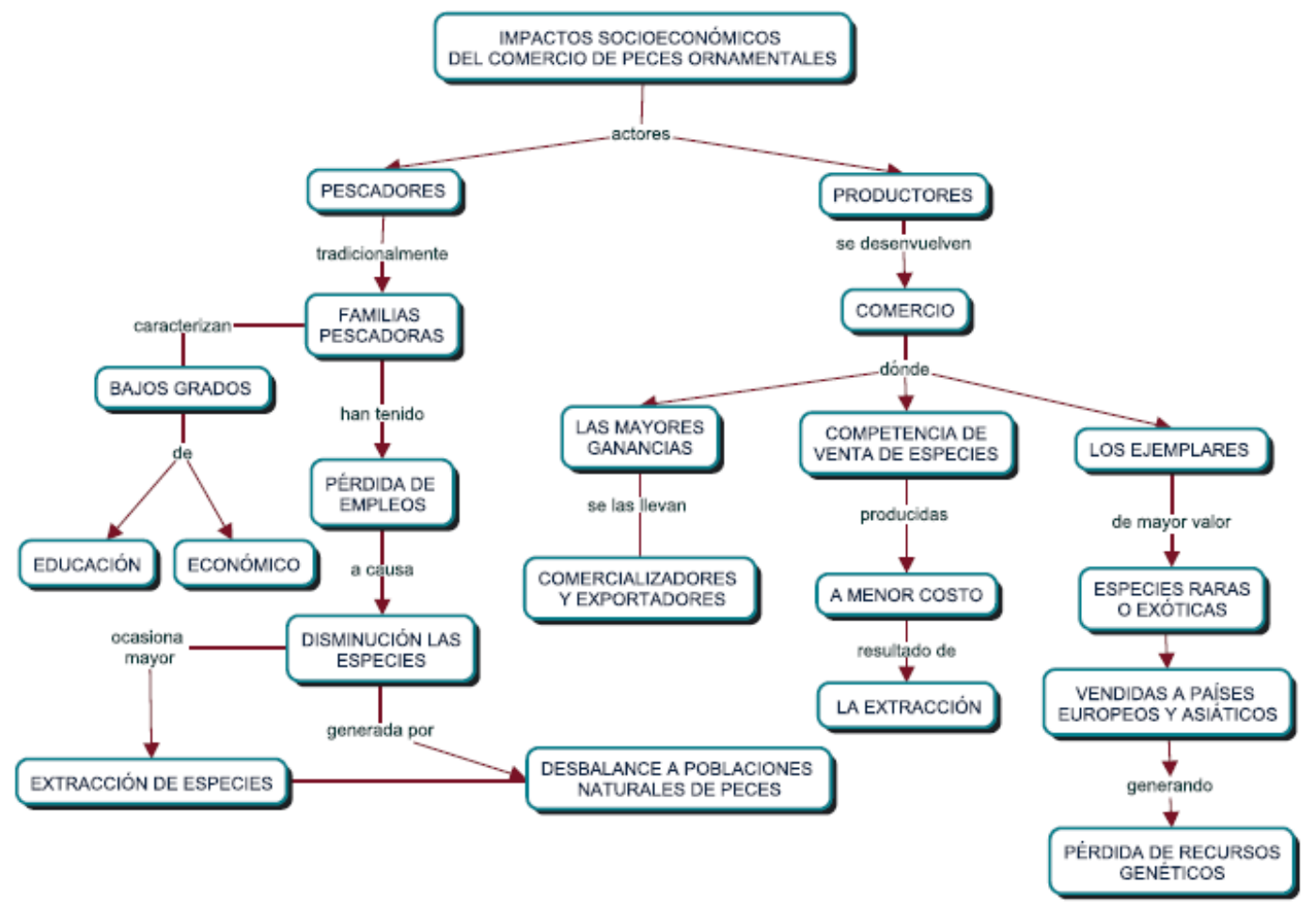

Fuente: elaboración propia.

En contraste, autores como Tlusty (2002) y Bunting y colaboradores (2013) consideran que, si se hace la pesca de una manera responsable y sostenible, la industria acuática puede ofrecer beneficios ecológicos y socioeconómicos, en la medida que se promuevan las buenas prácticas con el fin de mejorar los estándares y parámetros de la industria y que aseguren su aplicación, dando como resultado ganancias económicas y de bienestar para los peces.

Experiencias como las reportadas por Zuluaga y Franco (2013), en la Amazonia colombiana, a través de un trabajo con nueve comunidades en donde emplearon juegos económicos para formular o ajustar acuerdos de pesca efectivos y espacios de reflexión, permitieron confirmar e identificar las áreas clave de pesca dependiendo del ciclo hidrológico, las artes de pesca, las principales especies comercializadas y la generación de acuerdos de pesca sostenible para conservar los recursos pesqueros que utilizan para su sostenimiento.

Igualmente, en la Amazonia brasilera se identificaron experiencias de participación exitosa en el desarrollo de capacidades que deben ser consideradas como aporte a la sostenibilidad, cuyos resultados han ayudado a la certificación de indicaciones geográficas 'sellos verdes' a PO 'únicos en el mundo', que permiten la comercialización de forma legal y el beneficio a toda la cadena. Estos productos se comercializan en todos los mercados, según las normas de buenas prácticas de manejo en la captura y poscaptura. Adicionalmente, se proporcionaron servicios como rutas y circuitos turísticos con la oportunidad de dar a conocer a los turistas la cadena productiva de los PO, que incluye servicios como calidad, seguridad y confort (Chao y Prang, 1997; Schaefer, 2003; Munari, 2014).

En virtud de lo anterior, el comercio de $\mathrm{PO}$ en Colombia debe transitar a la mejora de sus prácticas de pesca y cultivo para contribuir con la conservación de las especies y ecosistemas, y con la creación de empleo y riqueza en las regiones que dependen de este recurso. Así mismo, es necesario fomentar el desarrollo y adopción de una cultura de buenas prácticas de cultivo, pesca y acopio, sobre la base de cuatro criterios principales como sugieren Vidal-Martínez et ál. (2017): la salud y el bienestar de los animales, la calidad de los alimentos suministrados, la integridad ambiental y la responsabilidad social que garanticen el desarrollo sustentable de la actividad y promuevan la recuperación del círculo vicioso de la sobreexplotación, degradación del ambiente y pobreza (figura 4). 
Figura 4. Recuperación del círculo vicioso de la sobreexplotación, degradación del ambiente y pobreza relacionada con el comercio de PO

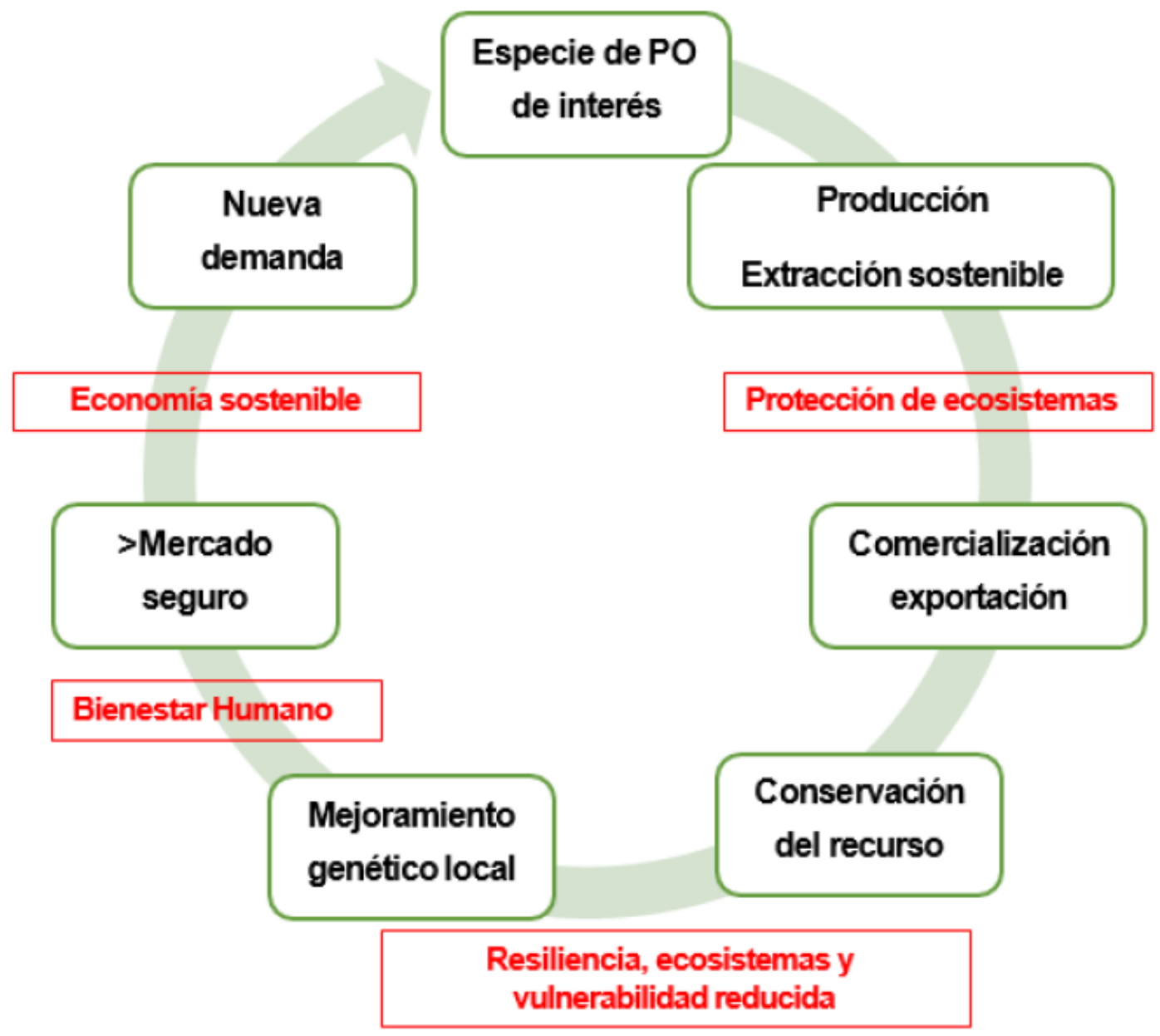

Fuente: elaboración propia.

\section{Conclusiones}

Debido a las prácticas actuales inadecuadas de pesca, se generan impactos negativos en los cuerpos de agua por el uso de productos químicos y explosivos; esto sumado a la extracción del recurso biológico, conlleva a desequilibrios poblacionales en los hábitats y a la disminución de los individuos objeto de pesca del cual depende el sustento económico de las familias ribereñas. Estas acciones ocasionan un círculo negativo continuo de extracción, pérdida del recurso biológico y afectación económica que direccionan a problemáticas sociales en las comunidades inherentes a la conocida trampa de la pobreza y medioambiente.

Los PO en el Colombia son una actividad prometedora para el sector agropecuario; sin embargo, es necesario que en el comercio se fomente el incentivo económico para los peces cultivados, ya que hoy es más rentable extraer que producir. Adicionalmente, existe en la cadena productiva un desvío del recurso económico que termina en los comercializadores y exportadores. No obstante, es necesario que se transite a sistemas sostenibles y que sean eficientes con el uso del agua, disminuyan la eutrofización y generen tratamientos terapéuticos alternativos con el fin de suprimir el uso de sustancias químicas.

También es importante que la academia, el sector gubernamental y el sector empresarial aúnan esfuerzos para incentivar el uso y la conservación de las especies ícticas nativas y endémicas, con el fin de conservar los recursos genéticos que hacen parte de la biodiversidad del país, junto con el desarrollo de buenas prácticas para la producción y comercialización de los PO.

El comercio de PO tiene beneficios directos e indirectos para las comunidades involucradas, así mismo puede servir para impulsar y promover la administración ambiental mediante la implementación de las buenas prácticas. Esto puede proporcionar fuertes incentivos para que las comunidades locales protejan los ecosistemas vulnerables y su competencia por el recurso.

Para optimizar los estándares y prácticas de la industria es perentorio que todas las partes interesadas trabajen en términos de responsabilidad y sostenibilidad: comunidades pesqueras, 
productoras, académicas, científicas, estatales y los demás actores de la cadena, en donde se expongan habilidades y experiencias sobre la mesa para desarrollar herramientas conjuntas que requiere la industria, y así hacerles frente a los desafíos que se avecinan.

La industria de PO necesita: inversión en infraestructura en áreas clave de recolección de peces, acortar las cadenas de suministro y proporcionar capacitación en cría moderna y gestión de asistencias técnicas. Para esto, las comunidades tienen un papel importante en la revisión crítica y la comunicación con la industria en cuanto a las mejoras que deben realizarse.

Se requiere de un trabajo educativo de responsabilidades con todos los participantes de la cadena en términos de conservación, bienestar animal y especies exóticas invasoras.

\section{Referencias}

Ajiaco-Martínez, R. E., Ramírez-Gil, H., Sánchez-Duarte, P., Lasso, C. A. y Trujillo, F. (2012). IV. Diagnóstico de la pesca ornamental en Colombia. En Reponame: Repositorio Instituto de Investigación de Recursos Biológicos Alexander von Humboldt. http://repository.humboldt.org.co/handle/20.50 $0.11761 / 31387$

Arboleda, D. (2006). Limnología aplicada a la acuicultura. REDVET. Revista Electrónica de Veterinaria, 7(11), 1-24. http s://www.redalyc.org/pdf/636/63612653022.pdf

Autoridad Nacional de Pesca y Acuicultura (Aunap). (2014). Plan Nacional para el Desarrollo de la Acuicultura Sostenible en Colombia \# PlaNDAS. Ministerio de agricultura y desarrollo rural \# MADR. https://www.aunap.gov.co/wp-content/uploads/2016/0 4/Plan-Nacional-para-el-Desarrollo-de-la-Acuicultura-Sosteni ble-Colombia.pdf

Badiola, M., Mendiola, D. y Bostock, J. (2012). Recirculating Aquaculture Systems (RAS) analysis: Main issues on management and future challenges. Aquacultural Engineering. 51, 26-35. https://doi.org/10.1016/j.aquaeng.2012.07.004

Barbier, E. B. (2010). 15 years of EDE - Advances and Challenges in Environment and Development Economics. Environment and Development Economics. 15(6), 635-660. https://doi.org/10.10 17/S1355770X1000032X

Barato, G., Penagos, G. y Iregui, C. (2012). Mapa epidemiológico de las enfermedades de los peces ornamentales en Colombia. Universidad Nacional de Colombia.

Borja, A. (2002). Los impactos ambientales de la acuicultura y la sostenibilidad de esta actividad. Boletín del Instituto Español de Oceanografía. 18(1-4), 41-49. https://core.ac.uk/download/pdf 171765141.pdf

Buschmann, A. H., López, D. A. y Medina, A. (1996). A review of the environmental effects and alternative production strategies of marine aquaculture in Chile. Aquacultural Engineering. 15(6), 397-421. https://doi.org/10.1016/S0144-8609(96)010060

Buschmann, A. y Fortt. A. (2005). Efectos Ambientales de la acuicultura intensiva y alternativas para un desarrollo sustentable. Revista Ambiente y Desarrollo de Cipma. 21(3), 58-64.

Bunting, S. W. (2013). Principles of sustainable aquaculture: promoting social, economic and environmental resilience. Earthscan/Routledge/Taylor \& Francis.
Cabello, F. C. (2006). Heavy use of prophylactic antibiotics in aquaculture: a growing problem for human and animal health and for the environment. Environmental Microbiology, 8(7), 1137-1144. https://pubmed.ncbi.nlm.nih.gov/16817922/

Cabrera, A. (2005). Situación del recurso peces ornamentales continentales en Venezuela [presentación de diapositivas]. Issuu. Dirección General de circuitos pesqueros y agrícolas. Ministerio de Agricultura y Tierras de la República Bolivariana de Venezuela. https://issuu.com/raysonarocha/docs/peces_orna mentales

Chao, N. L. y Prang, G. (1997). Project Piaba \# towards a sustainable ornamental fishery in the Amazon. Aquarium Sciences and Conservation, 1(2), 105-111. https://doi.org/10.1 023/A:1018339718743

Denommee-Patriganni, L. (2011). Évaluation de la performance des principaux éléments de gestion dans la durabilité des pêches artisanales des pays en développement [ensayo, Université de Sherbrooke]. https://savoirs.usherbrooke.ca/handle/11143/8 099

Dewi, R., Desrita, D. y Fadhilla, A. (2018). The prevalence of parasites in ornamental fish from fish market in Medan. IOP Conference Series: Earth and Environmental Science. 122, 012110. https://doi.org/10.1088/1755-1315/122/1/012110

Domínguez, L. M. y Botella, A. S. (2014). An overview of marine ornamental fish breeding as a potential support to the aquarium trade and to the conservation of natural fish populations. Sustainable Development and Planning. 9(4), 608-632. 10.2495/SDP-V9-N4-608-632

Espinosa, A. y Bermúdez, M. (2012). La acuicultura y su impacto al medio ambiente. Estudios Sociales, 2(12), 219-232. https://www.ciad.mx/archivos/revista-dr/RES_ESP2/ RES_Especial_2_10_Bermudez.pdf

García-Vásquez, A., Razo-Mendivil, U. y Rubio-Godoy, M. (2017). Triple trouble? Invasive poeciliid fishes carry the introduced tilapia pathogen Gyrodactylus cichlidarum in the Mexican highlands. Veterinary Parasitology. 235, 37-40. https ://doi.org/10.1016/j.vetpar.2017.01.014

Organización de las Naciones Unidas para la Alimentación y la Agricultura (FAO). (2005). Increasing the contribution of small.scale fisheries to poverty alleviation and food security (Technical Guidelines for Responsible Fisheries). http://www.f ao.org/3/a-a0237e.pdf

Organización de las Naciones Unidas para la Alimentación y la Agricultura (FAO). (2012). The state of world fisheries and aquaculture. Sustainability in action (Fisheries and Aquaculture Department). https://doi.org/10.4060/ca9229en

Organización de las Naciones Unidas para la Alimentación y la Agricultura (FAO). (2016). The state of world's fisheries and aquaculture 2016. Contributing to food security and nutrition for all (Fisheries and Aquaculture Department). http://www.fa o.org/3/a-i5798e.pdf

Folke, C. y Kautsky, N. (1989). The role of ecosystems for a sustainable development of aquaculture. Ambio. 18, 234-243.

García, A., Razo, U. y Rubio, M. (2017). Triple trouble? Invasive poeciliid fishes carry the introduced tilapia pathogen Gyrodactylus cichlidarum in the Mexican highlands. Veterinary Parasitology. 235, 37-40. 10.1016/j.vetpar.2017.01.014

Germain, N., Hartmann, H., Fernandez-Rivera, F. y Reyes, B. (2015). Ornamental reef fish fisheries: New indicators of sustainability and human development at a coastal community level. Ocean \& Coastal Management. 104, 136-149. https://doi .org/10.1016/j.ocecoaman.2014.12.007 
Guerrero, D., Franco-Jaramillo, M. y Rosell, J. (2017). The lack of alternative income sources: The case of ornamental fishing in the Inirida fluvial confluence, Colombian Amazon. Economía Agraria y Recursos Naturales. 17(2), 81-103. https://doi.org/10 .7201/earn.2017.02.04

Gasparini, J., Floeter, S., Ferreira, C. y Sazima, I. (2004). Marine Ornamental Trade in Brazil. Biodiversity \& Conservation. 14(12), 2883-2899. https://doi.org/10.1007/s10531-004-0222-1

Grant, M. J. y Booth, A. (2009). Una tipología de revisiones: un análisis de 14 tipos de revisión y metodologías asociadas. Revista de información y bibliotecas de salud. 26(2). 91-108.

Gräslund, S. y Bengtsson, B. (2001). Chemicals and Biological Products Used in SouthEast Asian Shrimp Farming and their Potential Impact on the Environment a Review. The Science of the Total Environment. 280(1-3), 93-131. 10.1016/ s0048-9697(01)00818-x

Guzmán-Maldonado, A. y Lasso, C. A. (2014). Análisis comparativo (1990-2014) de la pesquería de peces ornamentales en el departamento del Amazonas, Colombia. Biota Colombiana. 15(Supl. 1). http://revistas.humboldt.org.co/index .php/biota/article/view/344

Hoshino, E. de M., Hoshino, M. D. F. G. y Tavares-Dias, M. (2018). Parasites of ornamental fish commercialized in Macapá, Amapá State (Brazil). Revista Brasileira de Parasitologia Veterinária. 27(1), 74-79. https://dx.doi.org/10.1590/s1984-29 612018002

Ito, T., Kurita, J. y Haenen, O. L. M. (2017). Importation of CyHV-2-infected goldfish into the Netherlands. Diseases of Aquatic Organisms. 126(1), 51-62. https://doi.org/10.3354/dao 03157

Jaemwimol, P., Rawiwan, P., Tattiyapong, P., Saengnual, P. y Kamlangdee, A. (2018). Susceptibility of important warm water fish species to tilapia lake virus (TiLV) infection. Aquaculture. 497, 462-468. https://doi.org/10.1016/j.aquaculture.2018.08.02

King, T. (2019). Wild caught ornamental fish: A perspective from the UK ornamental aquatic industry on the sustainability of aquatic organisms and livelihoods. Journal of Fish Biology. 94(6), 925-936. https://doi.org/10.1111/jfb.13900

Ladisa, C., Bruni, M. y Lovatelli, A. (2017). Overview of ornamental species aquaculture. FAN - FAO Aquaculture Newsletter. 56, 39-40.

Mancera-Rodríguez, N. J. y Álvarez, R. (2008). Comercio de peces ornamentales en Colombia. Acta biológica colombiana. 13(1), 23-51.

Monteiro-Neto, C., De Andrade Cunha, F. E., Carvalho Nottingham, M., Araújo, M. E., Lucena Rosa, I. y Leite Barros, G. M. (2003). Analysis of the marine ornamental fish trade at Ceará State, northeast Brazil. Biodiversity \& Conservation. 12(6), 1287-1295. https://doi.org/10.1023/A:1023096023733

Moreno, F., Caro, C. I., Pinilla, G. A. y Osorio, D. P. (2017). Estado actual del conocimiento sobre microalgas del perifiton y macroinvertebrados bentónicos en el departamento del Meta, Colombia. Acta biológica de colombiana. 22(3), 274-306. http s://doi.org/10.15446/abc.v22n3.60619

Morales, J. y García, C. (2016). Estructura trófica de los peces en arroyos del Corral de San Luis, cuenca del Bajo Magdalena, Caribe, Colombia. Revista de Biología Tropical, 64(2), 715-732. https://doi.org/10.15517/rbt.v64i2.18781

Munari, M. (2014). Rota da piaba uma alternativa turística de sustentabilidade como resultado da indicao de procedencia do rio negro dos peixes ornamentais. Recsa, 3(2), 101-112. https:/ /revista.fisul.edu.br/index.php/revista/article/view/34/33

Ortega-Lara, A. (2016). Guía visual de los principales peces ornamentales continentales de Colombia. Serie Recursos Pesqueros de Colombia AUNAP. https://www.aunap.gov.co/wp-content/uploads/2016/ 10/GUIA-VISUAL-PECES-ORNAMENTALES-DE-COLOM BIA-2016.pdf

Passantino, A., Macri, D., Coluccio, P., Foti, F. y Marino, F. (2008). Importation of mycobacteriosis with ornamental fish: Medicolegal implications. Travel Medicine and Infectious Disease, 6(4), 240-244. https://doi.org/10.1016/j.tmaid.2007.12.003

Phitchaya, J., Pattarasuda, R., Puntanat, T., Pattrawut, S., Attapon, K. y Win, S. (2018). Susceptibility of important warm water fish species to tilapia lake virus (TiLV) infection. Author links open overlay panel Aquaculture. 497, 462-468. https://doi.org/10.10 16/j.aquaculture.2018.08.028

Ploeg, A. (2007). The Volume of the Ornamental Fish Trade. En A. Ploeg y Ornamental Fish International (organización), International transport of live fish in the ornamental aquatic industry. Ornamental Fish International (OFI).

Prada-Pedreros, S., Jhon G-F., Mondragón-Estupiñan, J. (2009). Capturas ícticas incidentales de la pesca ornamental en el período de aguas bajas en el área de influencia de Puerto Carreño, Orinoquia colombiana. Universitas Scientiarum. 14(2-3), 173-186. https://doi.org/10.11144/javeriana.SC14-2-3. ciid

Ramírez-Gil, H., Carrillo, L., Lacera, E. y Ajiaco, R. (2001). La pesca de especies de interés ornamental en el área de influencia de Puerto Carreño. En Ramírez-Gil. H. y Ajiaco-Martínez, R. H., La pesca en la Baja Orinoquia colombiana: pasado, presente y futuro. Instituto Nacional de Pesca y Acuicultura, INPA. http://localhost:8080/handle/11348/4972

Raghavan, R., Dahanukar, N., Tlusty, M., Rhyne, A., Kumar, K., Molur, S. y Rosser, A. (2013). Uncovering an obscure trade: Threatened freshwater fishes and the aquarium pet markets. Biological Conservation. 164, 158-169. https://doi.org/10.1016 /j.biocon.2013.04.019

Rhyne, A., Rotjan, R., Bruckner, A. y Tlusty, M. (2009). Crawling to collapse: ecologically unsound ornamental invertebrate fisheries. Plos One, 4(12), 8413. 10.1371/journal.pone.0008413

Rhyne, A., Tlusty, M., Schofield, P., Kaufman, L., Morris, J., Andrew, Jr. y Bruckner, W. (2012). Revealing the Appetite of the Marine Aquarium Fish Trade: The Volume and Biodiversity of Fish Imported into the United States. Aquarium Biodiversity, 7(5), 1-9. https://doi.org/10.1371/journal.pone.0035808

Rossoni, F., Ferreira, E. y Zuanon, J. (2014). A pesca eo conhecimento ecológico local dos pescadores de acará-disco (Symphysodon aequifasciatus, Pellegrin 1904: Cichlidae) na Reserva de Desenvolvimento Sustentável Piagaçu-Purus, baixo rio Purus, Brasil. Boletim do Museu Paraense Emílio Goeldi. Ciências Humanas, 9(1), 109-128. https://doi.org/10.1590/S19 81-81222014000100008

Sanna-Kaisa, J. y Jukka, S. (2004). Sustainable use of ornamental fish populations in Peruvian Amazonia. Iyonia, 7(2): 53-59. htt p://www.lyonia.org/downloadPDF.php?pdfID=2.269.1

Salazar, S., Pérez, J. y Alfonsi, C. (2008). Introducción y extracción de peces ornamentales y especies de invertebrados acuáticos en Venezuela. SABER. Revista Multidisciplinaria del Consejo de Investigación de la Universidad de Oriente. 20(2), 139-148.

Schaefer, A. (2003). Conservación y gestión de los recursos pesqueros ornamentales de la cuenca del río Negro, Amazonia, 
Brasil \# Proyecto Piaba, Copeia. 2003(3), 672-675. https://doi. org/10.1643/OT-03-017

Sobhee, S. K. (2004). Economic development, income inequality and environmental degradation of fisheries resources in Mauritius. Environment Manage. 34, 150-157. https://doi.org/1 0.1007/s00267-004-0133-7

Stern, N., Rachmilovitz, E. N., Sharon, G. y Diamant, A. (2018). The dire implications of releasing marine ornamental fishes into the wild: First reported case from the Red Sea. Marine Biodiversity. 48(3), 1615-1620. https://doi.org/10.1007/s12526 -016-0600-4

Stevens, C. H., Croft, D. P., Paull, G. C. y Tyler, C. R. (2017). Stress and welfare in ornamental fishes: What can be learned from aquaculture?: stress and welfare in ornamental fishes. Journal of Fish Biology. 91(2), 409-428. https://doi.org/10.1111/jfb.13377

Tlusty, M. F. (2002). The benefits and risks of aquaculture production for the aquarium trade. Aquaculture, 205, 203-219. https://doi.org/10.1016/S0044-8486(01)00683-4

Vaz, M., Rocha-Santos, T., Rocha, R., Lopes, I., Pereira, R., Duarte, A. C., Rubec, P. J. y Calado, R. (2012). Excreted thiocyanate detects live reef fishes illegally collected using cyanide - a non-invasive and non-destructive testing approach. Plos One, 1-7. https://doi.org/10.1371/journal.pone.0035355

Vergara, J., Haroun, R., González, M., Molina, L., Briz, M., Boyra, A., Gutiérrez, L. y Ballesta, A. (2005). Evaluación de impacto ambiental de acuicultura en jaulas en Canarias. Oceanográfica, Telde. https://www.oceanografica.com/descargas/Impactojaula s.pdf

Vidal-Martínez, V. M., Olvera-Novoa, M. A., Morales, V., CuéllarAnjel, J., Riofrío, A., Morales, R., Barato, P., Chávez, M. C., García, O. y Montoya, L. (2017). Manual de buenas prácticas de manejo para la piscicultura en agua dulce. OIRSA-OSPESCA, C.A. https://www.academia.edu/35667299/Manual_de_Buenas _Pr\%C3\%A1cticas_de_Manejo_para_la_Piscicultura_en_Agu a_Dulce.

Wood, E. (2001). Global Advances in Conservation and Management of Marine Ornamental Resources. Aquarium Sciences and Conservation, 3(1), 65-77. https://doi.org/10.102 3/A:1011391700880

Xu, K. X., Guo, M. H., Huang, Y. P., Li, X. D. y Sun, J. J. (2017). Rapid and sensitive detection of malachite green in aquaculture water by electrochemical preconcentration and surface-enhanced Raman scattering. Talanta, 180, 383-388. htt ps://doi.org/10.1016/j.talanta.2017.12.079

Zuluaga, P. A. y Franco-Jaramillo, M. (2013). Acuerdos de pesca sostenible en las comunidades de la Estrella Fluvial Inírida: Avances. ICAA. https://wwflac.awsassets.panda.org/download s/adpesca_baja_res.pdf

Zúñiga, P. T. (2010). Lineamientos de gestión ambiental para el control del tráfico ilícito de peces ornamentales dulceacuícolas de las cuencas Amazonas y Orinoco [tesis de maestría, Pontificia Universidad Javeriana]. https://repository.javeriana.e du.co/handle/10554/737

\section{Bibliografía consultada}

Fenner, D. (2012). Challenges for Managing Fisheries on Diverse Coral Reefs. Diversity, 4(1), 105-160. https://doi.org/10.3390/d 4010105 